\title{
The APC/C and CK1 in the developing brain
}

Editorial

\section{Clara Penas, Mary E. Hatten and Nagi G. Ayad}

Casein Kinase $1 \delta(\mathrm{CK} 1 \delta)$ is a serione/threonine kinase required for cell cycle progression, circadian rhythm, vesicle trafficking, and neurite outgrowth [1]. $\mathrm{CK} 1 \delta$ is also a therapeutic target in various cancers, Alzheimer's disease, alcoholism, and sleep disorders [1]. To examine the role of CK1 $\delta$ in brain development, we used cerebellar granule cell progenitors (GCPs) as a model system. GCPs are the most abundant neurons in the mammalian brain and are one of two principal neurons in the cerebellar circuitry [2]. CK1 $\delta$ is expressed in GCPs during peak times of proliferation (postnatal day 6-postnatal day 8). To probe a role for $\mathrm{CK} 1 \delta$ in proliferation of GCPs during this time, we assayed proliferation in GCPs lacking CK1 $\delta$, after knockdown of CK1 $\delta$ by RNAi methodology or in purified GCPs treated with highly specific CK1 $\delta$ inhibitor [3]. In all three cases, ${ }^{3} \mathrm{H}$-thymidine incorporation assays showed reduced levels of proliferation. Given CK1 1 's role in GCP neurogenesis, we anticipated that $\mathrm{CK} 1 \delta$ levels would decrease as GCPs exit the cell cycle. Indeed, we found that $\mathrm{CK} 1 \delta$ protein but not mRNA levels dropped during cell cycle exit, which suggested that CK1 $\delta$ is targeted for degradation during this time.

Importantly, biochemical assays demonstrate that $\mathrm{CK} 1 \delta$ is targeted for degradation via the Anaphase Promoting Complex/cyclosome (APC/C), a multisubunit E3 ubiquitin ligase, which has well-established roles in mitotic exit and G1 progression [2]. APC/C is also active in differentiating and differentiated cells [4]. $\mathrm{APC} / \mathrm{C}$ associates with one of two activators termed Cdc20 or Cdh1, which recruit substrates to bring them into close proximity of the E2 enzyme bound to APC/C [4]. We report that Cdh 1 binds to $\mathrm{CK} 1 \delta$ to initiate $\mathrm{APC} / \mathrm{C}$ dependent ubiqutination. In vitro ubiquitination assays containing purified $\mathrm{APC} / \mathrm{C}$ and $\mathrm{CK} 1 \delta$ demonstrate that $\mathrm{APC} / \mathrm{C}$ mediates $\mathrm{CK} 1 \delta$ polyubiquitination in vitro. $\mathrm{APC} / \mathrm{C}$ mediate ubiquitination of $\mathrm{CK} 1 \delta$ was dependent on two $\mathrm{N}$-terminal destruction boxes in $\mathrm{CK} 1 \delta$ as mutation of these sites abrogated ubiquitination in vitro [2]. To demonstrate a requirement for $\mathrm{CK} 1 \delta$ in vivo we deleted $\mathrm{CK} 1 \delta$ in GCPs in the cerebellum [2]. Deletion of the APC/C activator Cdh1 in GCPs increased CK1 $\delta$ levels, suggesting that CK1 $\delta$ is turned over in GCPs [2]. Collectively, these studies suggest that $\mathrm{APC} / \mathrm{C}$ targets $\mathrm{CK} 1 \delta$ for destruction in vitro and in vivo and that $\mathrm{APC} / \mathrm{C}^{\mathrm{Cdh} 1}$ is an important regulator of GCP proliferation by controlling $\mathrm{CK} 1 \delta$.

Our studies therefore suggest that APC/C-mediated degradation of $\mathrm{CK} 1 \delta$ functions in multiple steps in CNS neuronal differentiation. $\mathrm{CK} 1 \delta$ has been linked to neurite outgrowth [5] and thus it will be important to determine whether APC/C mediated degradation of $\mathrm{CK} 1 \delta$ occurs in axons or dendrites. Prior studies demonstrated that $\mathrm{APC} / \mathrm{C}$ inhibition in postmitotic neurons [4] increases neurite outgrowth while $\mathrm{CK} 1 \delta$ inhibition reduces neurite outgrowth in cell lines [5]. Thus, CK1 $\delta$ could be one of the substrates, which APC/C targets during neurite outgrowth, and whose levels rise during APC/C inhibition or depletion. It will be important to determine whether $\mathrm{CK} 1 \delta$ protein levels are modulated by $\mathrm{APC} / \mathrm{C}$ active in postmitotic neurons. Interestingly, there are two forms of the $\mathrm{APC} / \mathrm{C}$ that are active in postmitotic neurons, $\mathrm{APC} /$ $\mathrm{C}^{\mathrm{Cdh} 1}$ and $\mathrm{APC} / \mathrm{C}^{\mathrm{Cdc} 20}[4]$. $\mathrm{APC} / \mathrm{C}^{\mathrm{Cdh} 1}$ represses axonal growth [4] while $\mathrm{APC} / \mathrm{C}^{\mathrm{Cdc} 20}$ activity controls dendritic morphogenesis [4]. $\mathrm{APC} / \mathrm{C}^{\mathrm{Cdc} 20}$ is localized to centrosomes in postmitotic neurons. Given the finding that $\mathrm{CK} 1 \delta$ is localized to centrosomes [5] it will be interesting to determine whether $\mathrm{APC} / \mathrm{C}^{\mathrm{Cdc} 20}$ is able to induce $\mathrm{CK} 1 \delta$ destruction at centrosomes. An alternative could be that centrosome bound $\mathrm{CK} 1 \delta$ is protected from $\mathrm{APC} / \mathrm{C}$ mediated degradation as other $\mathrm{APC} / \mathrm{C}$ substrates cannot be ubiquitinated and degraded when bound to microtubules [6].

As centrosomal proteins often have roles in migration it will be important to determine whether
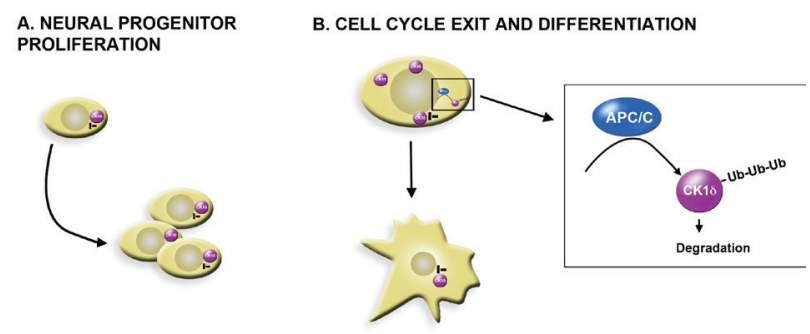

C. NEURITE OUTGROWTH AND MIGRATION

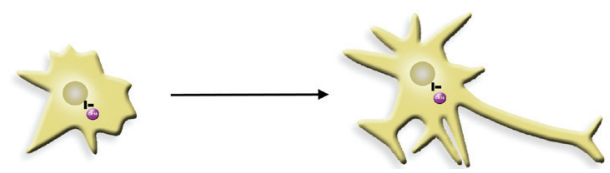

Figure 1: Model of $\mathrm{CK} 1 \delta$ and $\mathrm{APC} / \mathrm{C}$ during proliferation and differentiation of GCPs. A. CK $1 \delta$ activity is required for GCP proliferation. B. CK1 $\delta$ is degraded via $\mathrm{APC} / \mathrm{C}$ mediated ubiquitination during GCP cell cycle exit and differentiation. Some centrosome bound CK1 $\delta$ may be protected from APC/C mediated degradation. C. Centrosomal $\mathrm{CK} 1 \delta$ is required for neurite outgrowth and migration. 
$\mathrm{APC} / \mathrm{C}$ mediated control of $\mathrm{CK} 1 \delta$ is linked to migration of neuronal precursors. Consistent with a role for CK $1 \delta$ in neuronal migration we found that $\mathrm{CK} 1 \delta$ inhibition reduced GCP migration ex vivo (unpublished observations).

In addition, since $\mathrm{CK} 1 \delta$ has a role in ciliogenesis ${ }^{1}$ and $\mathrm{APC} / \mathrm{C}^{\mathrm{Cdc} 20}$ has been reported to be required for primary cilia formation [7], the APC/C may interact with $\mathrm{CK} 1 \delta$ in primary cilia. It will be interesting to determine whether $\mathrm{APC} / \mathrm{C}^{\mathrm{Cdc} 20}$ induces $\mathrm{CK} 1 \delta$ degradation within cilia. Interestingly, since the primary cilium is required for Hedgehog (Hh) pathway signaling as we found that CK1 $\delta$ inhibition or disruption reduced Hh signaling in GCPs [2], it will be essential to determine whether the $\mathrm{APC} / \mathrm{C}-\mathrm{CK} 1 \delta$ interaction is important for Hh signaling in GCPs. Future studies will determine the importance of the $\mathrm{APC} / \mathrm{C}-\mathrm{CK} 1 \delta$ interaction in various signaling pathways including $\mathrm{Hh}$ and WNT, where $\mathrm{CK} 1 \delta$ has been implicated [1]. Furthermore, it will be critical to determine whether the $\mathrm{APC} / \mathrm{C}-\mathrm{CK} 1 \delta$ interaction is dysregulated in various neurological diseases.

Nagi G. Ayad: Center for Therapeutic Innovation, Department of Psychiatry and Behavioral Sciences, University of Miami Miller School of Medicine, Miami, FL, USA

Correspondence to: Nagi G. Ayad, email nayad@miami. edu
Keywords: the anaphase promoting complex, neurite outgrowth, granule cell progenitors, casein kinase, cell cycle exit

Received: June 29, 2015

Published: July 09, 2015

\section{REFERENCES}

1. Knippschild U, et al. Frontiers in oncology. 2014; 4: 96.

2. Penas C, et al. Cell reports. 2015; 11: 249-60.

3. Penas C, et al. The Journal of biological chemistry. 2014; 289: 18893-903.

4. Penas C, et al. Frontiers in oncology. 2011; 1: 60.

5. Greer YE, et al. The Journal of cell biology. 2011; 192: $993-$ 1004.

6. $\quad$ Song L, et al. Molecular cell. 2014; 53: 179-92.

7. Wang W, et al. eLife 3. 2014; e0308. 